\title{
On Kleene Algebras and Closed Semirings*
}

\author{
Dexter Kozen ${ }^{\dagger}$ \\ Department of Computer Science \\ Cornell University \\ Ithaca, New York 14853-7501, USA
}

May 31, 1990

\begin{abstract}
Kleene algebras are an important class of algebraic structures that arise in diverse areas of computer science: program logic and semantics, relational algebra, automata theory, and the design and analysis of algorithms. The literature contains several inequivalent definitions of Kleene algebras and related algebraic structures [2, 14, 15, $5,6,1,10,7]$.

In this paper we establish some new relationships among these structures. Our main results are:

- There is a Kleene algebra in the sense of [6] that is not *-continuous.

- The categories of *-continuous Kleene algebras [5, 6], closed semirings $[1,10]$ and S-algebras [2] are strongly related by adjunctions.

- The axioms of Kleene algebra in the sense of [6] are not complete for the universal Horn theory of the regular events. This refutes a conjecture of Conway [2, p. 103].

- Right-handed Kleene algebras are not necessarily left-handed Kleene algebras. This verifies a weaker version of a conjecture of Pratt [15].
\end{abstract}

*In Rovan, ed., Proc. Math. Found. Comput. Sci., volume 452 of Lect. Notes in Comput. Sci., pages 26-47. Springer, 1990.

${ }^{\dagger}$ Supported by NSF grant CCR-8901061. 


\section{Introduction}

Kleene algebras are algebraic structures with operators $+, \cdot,{ }^{*}, 0$, and 1 satisfying certain properties. They have been used to model programs in Dynamic Logic [14, 5], to prove the equivalence of regular expressions and finite automata [2, 1], to give fast algorithms for transitive closure and shortest paths in directed graphs [1,10], and to axiomatize relational algebra $[11,12,15]$.

There has been some disagreement regarding the proper definition of Kleene algebras. At least ten inequivalent definitions of Kleene algebras or related structures have appeared in the literature $[2,14,15,5,6]$, all serving roughly the same purpose. It is important to understand the relationships between these classes in order to extract the axiomatic essence of Kleene algebra. Perhaps it is not too unreasonable to hope that we might one day converge on a standard definition.

In this paper we discuss some of the relationships among these classes. For the purposes of this paper, we adopt the definition of [6], which defines a Kleene algebra to be an idempotent semiring with a ${ }^{*}$ operator satisfying

$$
\begin{aligned}
1+a a^{*} & \leq a^{*} \\
1+a^{*} a & \leq a^{*} \\
a x \leq x & \rightarrow a^{*} x \leq x \\
x a \leq x & \rightarrow x a^{*} \leq x
\end{aligned}
$$

where $\leq$ refers to the natural order in the idempotent semiring. It is shown in [6] that these axioms completely characterize the algebra of regular events, giving a finitary axiomatization of that equational theory and thus improving Salomaa's completeness theorem [16]. A similar (and in fact stronger) theorem is stated by Conway without proof [2, p. 108]; but to his knowledge [3] and the author's, no proof has ever appeared in the literature.

A Kleene algebra is called ${ }^{*}$-continuous if it satisfies the axiom

$$
a b^{*} c=\sum_{n} a b^{n} c
$$

where $\sum$ refers to the supremum in the natural order $\leq$. This infinitary property of * implies the previous four. These algebras have been used to model programs in Dynamic Logic [5].

In the design and analysis of algorithms, a related family of structures called closed semirings form an important algebraic abstraction. They give a unified framework for deriving efficient algorithms for transitive closure and all-pairs shortest paths in graphs and constructing regular expressions from finite automata [1, 10]. Very fast algorithms for all these problems can be derived as special cases of a single general algorithm over an arbitrary closed semiring. Closed semirings are defined in terms of a countable summation operator $\sum$ as well as $\cdot, 0$, and 1 ; the operator ${ }^{*}$ is defined in terms of $\sum$. Under the operations of 
(finite) $+, \cdot,^{*}, 0$, and 1 , any closed semiring is a ${ }^{*}$-continuous Kleene algebra. In fact, in the treatment of $[1,10]$, the sole purpose of $\sum$ seems to be to define ${ }^{*}$. A more descriptive name for closed semirings might be $\omega$-complete idempotent semirings.

These algebras are strongly related to several classes of algebras defined by Conway in his 1971 monograph [2]. Conway's S-algebras are similar to closed semirings, except that arbitrary sums, not just countable ones, are permitted. A better name for $\mathbf{S}$-algebras might be complete idempotent semirings. The operation ${ }^{*}$ is defined as in closed semirings in terms of $\sum$, and again this seems to be the sole purpose of $\sum$. The $\mathbf{N}$-algebras are algebras of signature $\left(+, \cdot,{ }^{*}, 0,1\right)$ that are subsets of $\mathbf{S}$-algebras containing 0 and 1 and closed under (finite),$+ \cdot$, and ${ }^{*}$. Combining results of [5] and [2, Theorem 1, p. 102], it can be shown that the classes of $\mathbf{N}$-algebras and ${ }^{*}$-continuous Kleene algebras coincide. An $\mathbf{R}$-algebra is any algebra of signature $\left(+, \cdot,{ }^{*}, 0,1\right)$ satisfying the equational theory of the $\mathbf{N}$-algebras.

In $[14,15]$, Pratt gives two definitions of Kleene algebras in the context of dynamic algebra. In [14], Kleene algebras are defined to be the Kleenean component of the separable dynamic algebras; in [15], this class is enlarged to contain all subalgebras of such algebras.

Generalizations of Kleene's and Parikh's Theorems have been given by Kuich [7] in $\ell$ complete semirings, which are similar to $\mathbf{S}$-algebras in all respects except that idempotence of $\sum$ is replaced by a weaker condition called $\ell$-completeness.

In this paper we establish some new relationships among some of these structures. Our main results are as follows:

- It is known that the $\mathbf{R}$-algebras, Kleene algebras, ${ }^{*}$-continuous Kleene algebras (a.k.a. $\mathbf{N}$-algebras), closed semirings, and $\mathbf{S}$-algebras each contain the next in the list. All inclusions were known to be strict except Kleene algebras and ${ }^{*}$-continuous Kleene algebras. We complete the picture by constructing a Kleene algebra that is not * continuous. Such an algebra must necessarily be infinite, since all finite Kleene algebras are ${ }^{*}$-continuous.

- Conway gives a construction that embeds every ${ }^{*}$-continuous Kleene algebra in an $\mathbf{S}$ algebra [2, Theorem 1, p. 102]. This construction can be described as a completion by ${ }^{*}$-ideals. We show that this construction extends to a functor from the category $\mathbf{K A}^{*}$ of ${ }^{*}$-continuous Kleene algebras and Kleene algebra morphisms to the category SA of S-algebras and continuous semiring morphisms, and that this functor is a left adjoint for the forgetful functor that assigns to each $\mathbf{S}$-algebra its Kleene algebra structure. Moreover, this adjunction factors into two adjunctions, one relating $\mathbf{K A}^{*}$ and the category $\mathbf{C S}$ of closed semirings and $\boldsymbol{\omega}$-continuous semiring morphisms, and one relating $\mathbf{C S}$ and $\mathbf{S A}$. The functor from $\mathbf{K A}^{*}$ to $\mathbf{C S}$ can be described as a completion by countably generated ${ }^{*}$-ideals.

- We show that the axioms for Kleene algebra [6] are not complete for the universal Horn theory of the regular events. This refutes a conjecture of Conway [2, p. 103]. 
- We show that right-handed Kleene algebras do not have to be left-handed. This verifies a weaker version of a conjecture of Pratt [15]. Pratt's conjecture referred to Boolean semirings (yet another related structure) in which the natural order $\leq$ comes from a Boolean algebra.

\section{Definitions}

\subsection{Kleene Algebras}

For the purposes of this paper, we adopt the definition of [6]. There, a Kleene algebra is defined to be a structure $\mathcal{K}$ with binary operations + and $\cdot$, unary operation ${ }^{*}$, and constants 0 and 1 such that

$$
(\mathcal{K},+, \cdot, 0,1)
$$

is an idempotent semiring, i.e.

- + is associative, commutative, and idempotent $(a+a=a)$ with identity 0

- . is associative with two-sided identity 1

- distributes over + on both sides; and

- 0 is a two-sided annihilator for .

and the operation * satisfies the following properties:

$$
\begin{aligned}
1+a a^{*} & \leq a^{*} \\
1+a^{*} a & \leq a^{*} \\
a x \leq x & \rightarrow a^{*} x \leq x \\
x a \leq x & \rightarrow x a^{*} \leq x
\end{aligned}
$$

where $\leq$ refers to the natural partial order on $\mathcal{K}:$

$$
a \leq b \quad \leftrightarrow \quad a+b=b
$$

We often abbreviate $a \cdot b$ to $a b$, and avoid parentheses by assigning the priority ${ }^{*}>\cdot>+$ to the operators.

A somewhat stronger axiom for ${ }^{*}$ is the so-called ${ }^{*}$-continuity condition:

$$
a b^{*} c=\sum_{n \geq 0} a b^{n} c
$$


where $b^{0}=1, b^{n+1}=b b^{n}$, and $\sum$ refers to the supremum with respect to the natural order $\leq$. A Kleene algebra satisfying $(5)$ is said to be ${ }^{*}$-continuous. In any idempotent semiring, (5) implies (1-4).

An idempotent semiring satisfying (1-3) is called a right-handed Kleene algebra. An idempotent semiring satisfying (1-2) and (4) is called a left-handed Kleene algebra. Thus an idempotent semiring is a Kleene algebra iff it is both left- and right-handed.

The category of Kleene algebras and Kleene algebra homomorphisms will be denoted KA. The full subcategory of ${ }^{*}$-continuous Kleene algebras will be denoted $\mathbf{K A}^{*}$.

\subsubsection{Examples of Kleene Algebras}

An important example of a Kleene algebra is $\mathbf{R e g}_{\Sigma}$, the family of regular sets over a finite alphabet $\Sigma$. The equational theory of this structure is called the algebra of regular events. This theory was axiomatized by Salomaa [16], but his axiomatization involved rules that were not sound in general when interpreted over other Kleene algebras. It was shown in [6] that the Kleene algebra axioms completely axiomatize the algebra of regular events. In other words, $\boldsymbol{R e g}_{\Sigma}$ is the free Kleene algebra on free generators $\Sigma$. Conway [2, Theorem 5 , p. 108] claimed without proof that the axioms for right-handed Kleene algebra completely axiomatize the algebra of regular events, but to his knowledge [3] and the author's, no proof has ever appeared in the literature. A weaker completeness proof involving (5) instead of (1-4) was previously given in [5].

Another important class of Kleene algebras is the class of algebras of binary relations. A family of binary relations with operations of $\cup$ for + , relational composition

$$
R \circ S=\{(x, z) \mid \exists y(x, y) \in R \text { and }(y, z) \in S\}
$$

for $\cdot$, the empty relation for 0 , the identity relation for 1 , and reflexive transitive closure for

$*$ is a Kleene algebra. Kleene algebras of binary relations are used to model programs in Dynamic Logic and other logics of programs [4, 13].

All these examples are ${ }^{*}$-continuous. In $\S 3$ we will construct a Kleene algebra that is not *-continuous.

The $n \times n$ matrices over a Kleene algebra again form a Kleene algebra [6].

\subsubsection{Elementary Properties of Kleene Algebras}

We list here some elementary consequences of the Kleene algebra axioms. See [2, 15, 6] for proofs.

In any Kleene algebra $\mathcal{K}$, the relation $\leq$ is a partial order, and $\mathcal{K}$ is an upper semilattice with join + and minimum element 0 . Also,

$$
a \leq b \quad \rightarrow \quad a c \leq b c
$$




$$
\begin{aligned}
a \leq b & \rightarrow c a \leq c b \\
a \leq b & \rightarrow a+c \leq b+c \\
a \leq b & \rightarrow a^{*} \leq b^{*} \\
1+a+a^{*} a^{*} & =a^{*} \\
a^{* *} & =a^{*} \\
0^{*} & =1 \\
1+a a^{*} & =a^{*} \\
1+a^{*} a & =a^{*} \\
b+a x \leq x & \rightarrow a^{*} b \leq x \\
b+x a \leq x & \rightarrow b a^{*} \leq x \\
a x=x b & \rightarrow a^{*} x=x b^{*} \\
(c d)^{*} c & =c(d c)^{*} \\
(a+b)^{*} & =a^{*}\left(b a^{*}\right)^{*} \\
p p^{\prime}=1 \wedge p^{\prime} p=1 & \rightarrow p^{\prime} a^{*} p=\left(p^{\prime} a p\right)^{*} .
\end{aligned}
$$

\subsection{Closed Semirings}

According to $[1,10]$, a closed semiring is an idempotent semiring equipped with an infinitary summation operator $\sum$ that applies to countable sequences and satisfies infinitary associativity, commutativity, idempotence, and distributivity laws. (In [1, 10] both the finitary and infinitary summation operators + and $\sum$ are taken as primitive, and no explicit axioms connecting them are given. However, it is clear from subsequent arguments what was meant and how to complete the axiomatization.)

Since $\sum$ is associative, commutative, and idempotent, its value on a given sequence is independent of the order and multiplicity of elements occurring in the sequence. Thus we might as well define $\sum$ on finite or countable subsets instead of sequences. In this view, $\sum$ gives the supremum with respect to the natural order $\leq$. To see this, let $A$ be a nonempty finite or countable subset of a closed semiring $C$. If $x \in A$, then

$$
\begin{aligned}
x+\sum A & =\sum(A \cup\{x\}) \\
& =\sum A,
\end{aligned}
$$

thus $x \leq \sum A$; and if $x \leq y$ for all $x \in A$, then $x+y=y$ for all $x \in A$, thus

$$
\begin{aligned}
\left(\sum A\right)+y & =\left(\sum_{x \in A} x\right)+\left(\sum_{x \in A} y\right) \\
& =\sum_{x \in A}(x+y)
\end{aligned}
$$




$$
\begin{aligned}
& =\sum_{x \in A} y \\
& =y,
\end{aligned}
$$

therefore

$$
\sum A \leq y
$$

Thus we may regard $\sum$ as a supremum operator for countable sets. The infinite distributivity laws then say exactly that $\cdot$ is bicontinuous with respect to countable suprema. Note also that 0 is the supremum of the empty set.

In a closed semiring $C$, one can define ${ }^{*}$ by:

$$
b^{*}=\sum_{n \leq \omega} b^{n}
$$

where $b^{0}=1$ and $b^{n+1}=b b^{n}$. Then by infinite distributivity,

$$
a b^{*} c=\sum_{n} a b^{n} c,
$$

therefore $\mathcal{C}$ is also a ${ }^{*}$-continuous Kleene algebra $\mathbf{K} C$. Moreover, if $C$ and $C^{\prime}$ are two closed semirings and $h: C \rightarrow C^{\prime}$ is an $\omega$-continuous semiring morphism (i.e., a semiring morphism that preserves suprema of countable sets), then $h$ must preserve ${ }^{*}$, therefore constitutes a Kleene algebra morphism $\mathbf{K} h: \mathbf{K} C \rightarrow \mathbf{K} C^{\prime}$. We thus have a forgetful functor

$$
\mathbf{K}: \mathbf{C S} \rightarrow \mathbf{K A}^{*}
$$

from the category $\mathbf{C S}$ of closed semirings and $\omega$-continuous semiring morphisms to the category $\mathbf{K A}^{*}$ of ${ }^{*}$-continuous Kleene algebras and Kleene algebra morphisms. We will show in $\S 4$ that this functor has a left adjoint.

\subsubsection{Examples of Closed Semirings}

The regular sets $\mathbf{R e g}_{\Sigma}$ do not form a closed semiring: if $A$ is nonregular, the countable set $\{\{x\} \mid x \in A\}$ has no supremum. However, the power set of $\Sigma^{*}$ does form a closed semiring.

Similarly, the family of all binary relations on a set forms a closed semiring under the relational operations described in $\$ 2.1 .1$ and infinite union for $\sum$.

A rather bizarre example of a closed semiring is the so-called $(\mathrm{min},+)$ algebra, used to derive an efficient algorithm for finding the shortest paths between all pairs of vertices in a directed graph $[1,10]$. This algebra consists of the nonnegative reals with an infinite element $\infty$ adjoined. The Kleene algebra operations $+, \cdot, 0$, and 1 are given by $\min ,+, \infty$, and 0 , respectively. The operation $*$ is the constant 0 function.

As with Kleene algebras, the $n \times n$ matrices over a closed semiring again form a closed semiring. 


\subsection{Conway's Algebras}

According to the definition in [2], an $\mathbf{S}$-algebra

$$
\left(\mathcal{S}, \sum, \cdot 0,1\right)
$$

is similar to a closed semiring, except that $\sum$ is defined not on sequences but on multisets of elements of $\mathcal{S}$; moreover, there is no cardinality restriction on the multiset (thus $\sum$ is too big to be represented in ZF set theory!). However, as with closed semirings, the value that $\sum$ takes on a given multiset is independent of the multiplicity of the elements, so $\sum$ might as well be defined on subsets of $\mathcal{S}$ instead of multisets. So defined, $\sum A$ gives the supremum of $A$ with respect to the order $\leq$. (We assume the axiom $\sum\{a\}=a$, which is omitted in [2].)

Thus, the only essential difference between $\mathbf{S}$-algebras and closed semirings is that closed semirings are only required to contain suprema of countable sets, whereas $\mathbf{S}$-algebras must contain suprema of all sets. Thus every $\mathbf{S}$-algebra is automatically a closed semiring and every continuous semiring morphism (semiring morphism preserving all suprema) is automatically $\omega$-continuous, and these notions coincide on countable algebras. This gives a forgetful functor $\mathbf{G}: \mathbf{S A} \rightarrow \mathbf{C S}$ from the category $\mathbf{S A}$ of $\mathbf{S}$-algebras and continuous semiring morphisms to CS.

\section{A Kleene algebra that is not ${ }^{*}$-continuous}

Recall the definitions of the categories

\begin{tabular}{lll} 
category & objects & morphisms \\
\hline SA & S-algebras & continuous semiring morphisms \\
CS & closed semirings & $\omega$-continuous semiring morphisms \\
KA ${ }^{*}$ & ${ }^{*}$-continuous Kleene algebras & Kleene algebra morphisms \\
KA & Kleene algebras & Kleene algebra morphisms \\
RA & R-algebras & maps preserving $+, \cdot,{ }^{*}, 0,1$
\end{tabular}

From results of $[2,6,5]$, the equational theories of all these algebras coincide. The following inclusions are also known:

$$
\mathbf{S A} \subseteq \mathbf{C S} \subseteq \mathbf{K A}^{*} \subseteq \mathbf{K A} \subseteq \mathbf{R A}
$$

All inclusions were known to be strict except $\mathbf{K A}^{*} \subseteq \mathbf{K A}$. Conway [2, p. 102] gives a four-element $\mathbf{R}$-algebra $R_{4}$ that is not a ${ }^{*}$-continuous Kleene algebra. It is easily shown that all finite Kleene algebras are ${ }^{*}$-continuous, thus $R_{4}$ is not a Kleene algebra either. The family $\mathbf{R e g}_{\Sigma}$ of regular events over an alphabet $\Sigma$ gives an example of a ${ }^{*}$-continuous Kleene algebra that is not a closed semiring, as shown in $\$ 2.2 .1$. It is also easy to construct closed 
semirings that are not $\mathbf{S}$-algebras; for example, the countable and co-countable subsets of $\omega_{1}$ (the first uncoutable ordinal) with operations of set union for $\sum$, set intersection for $\cdot \emptyset$ for $0, \omega_{1}$ for 1 , and $A^{*}=\omega_{1}$.

To complete the picture, we need to construct a Kleene algebra that is not ${ }^{*}$-continuous. Let $\omega^{2}$ denote the set of ordered pairs of natural numbers and let $\perp$ and $T$ be new elements. Order these elements such that $\perp$ is the minimum element, $T$ is the maximum element, and $\omega^{2}$ is ordered lexicographically in between. Define + to give the supremum in this order. Define $\cdot$ according to the following table:

$$
\begin{aligned}
x \cdot \perp & =\perp \cdot x=\perp \\
x \cdot \top & =\top \cdot x=\top, x \neq \perp \\
(a, b) \cdot(c, d) & =(a+c, b+d) .
\end{aligned}
$$

Then $\perp$ is the additive identity and $(0,0)$ is the multiplicative identity. Finally, define

$$
a^{*}= \begin{cases}(0,0), & \text { if } a=\perp \text { or } a=(0,0) \\ \top, & \text { otherwise. }\end{cases}
$$

It is easily checked that this is a Kleene algebra. We verify the axiom

$$
a x \leq x \rightarrow a^{*} x \leq x
$$

explicitly. Assuming $a x \leq x$, we wish to show $a^{*} x \leq x$. If $a=\perp$ or $a=(0,0)$, then $a^{*}=(0,0)$ and we are done, since $(0,0)$ is the multiplicative identity. If $x=\perp$ or $x=\mathrm{\top}$, we are done. Otherwise, $a>(0,0)$ and $x=(u, v)$, in which case $a x>x$, contradicting the assumption.

This Kleene algebra is not ${ }^{*}$-continuous, since $(0,1)^{*}=T$, but

$$
\sum_{n}(0,1)^{n}=\sum_{n}(0, n)=(1,0) .
$$

\section{Some categorical connections}

In this section we establish strong relationships among the categories $\mathbf{K A}^{*}, \mathbf{C S}$, and $\mathbf{S A}$. Recall from $\S 3$ that $\mathbf{K A}$ is the category of Kleene algebras and Kleene algebra morphisms; $\mathbf{K A}^{*}$ is the full subcategory of ${ }^{*}$-continuous Kleene algebras and Kleene algebra morphisms; CS is the category of closed semirings and $\omega$-continuous semiring morphisms; and $\mathbf{S A}$ is the category of $\mathbf{S}$-algebras and continuous semiring morphisms.

In [2, Theorem 1, p. 102], Conway gives a construction that shows that every ${ }^{*}$-continuous Kleene algebra is embedded in an $\mathbf{S}$-algebra. This construction can be described as a completion by ${ }^{*}$-ideals. Conway gives the construction but omits the proof, claiming that the 
"details are rather subtle." To his knowledge [3] and the author's, no proof has appeared in the literature. We will show in this section that Conway's construction constitutes a functor $\mathbf{K A}^{*} \rightarrow \mathbf{S A}$ that is a left adjoint for the forgetful functor $\mathbf{S A} \rightarrow \mathbf{K A} \mathbf{A}^{*}$. Moreover, this adjunction factors into a composition of adjunctions

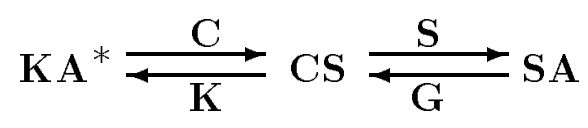

where $\mathbf{C}: \mathbf{K A}^{*} \rightarrow \mathbf{C S}$ is a left adjoint for the forgetful functor $\mathbf{K}: \mathbf{C S} \rightarrow \mathbf{K A}^{*}$ and $\mathbf{S}: \mathbf{C S} \rightarrow \mathbf{S A}$ is a left adjoint for the forgetful functor $\mathbf{G}: \mathbf{S A} \rightarrow \mathbf{C S}$; Conway's construction is then the composition $\mathbf{S} \circ \mathbf{C}$.

The construction $\mathbf{C}$, which shows that every ${ }^{*}$-continuous Kleene algebra is embedded in a closed semiring, can be described as a completion by countably generated ${ }^{*}$-ideals.

\subsection{Kleene algebras and closed semirings}

Definition 1 (Conway [2]) Let $\mathcal{K}$ be a ${ }^{*}$-continuous Kleene algebra. $A^{*}$-ideal is a subset $A$ of $\mathcal{K}$ such that

- $A$ is nonempty

- $A$ is closed under +

- $A$ is closed downward under $\leq$

- if $a b^{n} c \in A$ for all $n \geq 0$, then $a b^{*} c \in A$.

We say that a nonempty set $A$ generates a ${ }^{*}$-ideal $I$ if $I$ is the smallest ${ }^{*}$-ideal containing $A$. We write $\langle A\rangle$ to denote the ${ }^{*}$-ideal generated by $A$. A ${ }^{*}$-ideal is countably generated if it has a countable generating set. If $A$ is a singleton $\{x\}$, then we abbreviate $\langle\{x\}\rangle$ by $\langle x\rangle$. Such an ideal is called principal with generator $x$.

Let $\mathcal{K}$ be a ${ }^{*}$-continuous Kleene algebra. For subsets $A, B \subseteq \mathcal{K}$, define

$$
\begin{aligned}
A \oplus B & =\{a+b \mid a \in A, b \in B\} \\
A \odot B & =\{a \cdot b \mid a \in A, b \in B\} \\
A \downarrow & =\{y \mid \exists x \in A y \leq x\} \\
A_{*} & =\left\{a b^{*} c \mid a b^{n} c \in A \text { for all } n \geq 0\right\} .
\end{aligned}
$$

Note that for principal ideals,

$$
\langle x\rangle=\{x\} \downarrow
$$


Note also that

$$
\begin{aligned}
A \odot(B \oplus C) & \subseteq(A \odot B) \oplus(A \odot C) \\
(A \oplus B) \odot C & \subseteq(A \odot C) \oplus(B \odot C) \\
A \odot(B \downarrow) & \subseteq(A \odot B) \downarrow \\
(A \downarrow) \odot B & \subseteq(A \odot B) \downarrow \\
A \odot\left(B_{*}\right) & \subseteq(A \odot B)_{*} \\
\left(A_{*}\right) \odot B & \subseteq(A \odot B)_{*} .
\end{aligned}
$$

Define the set operator $\tau$ on nonempty subsets $A \subseteq \mathcal{K}$ as follows:

$$
\tau(A)=(A \oplus A) \cup A \downarrow \cup A_{*} .
$$

Then $A$ is a ${ }^{*}$-ideal iff it is nonempty and closed under $\tau$. Note that $A \subseteq \tau(A)$ and that $\tau$ is monotone in the sense that if $A \subseteq B$ then $\tau(A) \subseteq \tau(B)$. Using $\tau$, define for any $A \subseteq \mathcal{K}$ the transfinite sequence

$$
\begin{aligned}
\tau^{0}(A) & =A \\
\tau^{\alpha+1}(A) & =\tau\left(\tau^{\alpha}(A)\right) \\
\tau^{\lambda}(A) & =\bigcup_{\alpha<\lambda} \tau^{\alpha}(A), \quad \lambda \text { a limit ordinal } \\
\tau^{*}(A) & =\bigcup_{\alpha} \tau^{\alpha}(A) .
\end{aligned}
$$

Note that $\tau^{*}(A)=\tau^{\kappa}(A)$ where $\kappa$ is the least ordinal such that $\tau^{\kappa+1}(A)=\tau^{\kappa}(A)$. Such a $\kappa$ exists since $\tau$ is monotone. In fact, it can be shown that $\kappa \leq \omega_{1}$, the first uncountable ordinal. By the Knaster-Tarski Theorem,

$$
\tau^{*}(A)=\langle A\rangle \text {. }
$$

\section{Lemma 2}

$$
\begin{aligned}
\tau(A) \odot B & \subseteq \tau(A \odot B) \\
A \odot \tau(B) & \subseteq \tau(A \odot B) .
\end{aligned}
$$

Proof. We prove the first statement; the second is symmetric.

$$
\begin{aligned}
\tau(A) \odot B & =\left((A \oplus A) \cup A \downarrow \cup A_{*}\right) \odot B \\
& =((A \oplus A) \odot B) \cup(A \downarrow \odot B) \cup\left(A_{*} \odot B\right) \\
& \subseteq((A \odot B) \oplus(A \odot B)) \cup(A \odot B) \downarrow \cup(A \odot B)_{*} \\
& =\tau(A \odot B) .
\end{aligned}
$$




\section{Lemma 3}

$$
\langle A \odot B\rangle=\langle\langle A\rangle \odot\langle B\rangle\rangle
$$

Proof. The inclusion $\subseteq$ follows from monotonicity. For the reverse inclusion, we prove by transfinite induction that for all ordinals $\alpha, \beta$,

$$
\tau^{\alpha}(A) \odot \tau^{\beta}(B) \subseteq\langle A \odot B\rangle
$$

Certainly

$$
\tau^{0}(A) \odot \tau^{0}(B)=A \odot B \subseteq\langle A \odot B\rangle
$$

Also,

$$
\begin{aligned}
\tau^{\alpha+1}(A) \odot \tau^{\beta}(B) & =\tau\left(\tau^{\alpha}(A)\right) \odot \tau^{\beta}(B) \\
& \subseteq \tau\left(\tau^{\alpha}(A) \odot \tau^{\beta}(B)\right) \text { by Lemma } 2 \\
& \subseteq \tau(\langle A \odot B\rangle) \text { by induction hypothesis and monotonicity of } \tau \\
& =\langle A \odot B\rangle
\end{aligned}
$$

and similarly,

$$
\tau^{\alpha}(A) \odot \tau^{\beta+1}(B) \subseteq\langle A \odot B\rangle
$$

For limit ordinals $\lambda$,

$$
\begin{aligned}
\tau^{\lambda}(A) \odot \tau^{\beta}(B) & =\left(\bigcup_{\alpha<\lambda} \tau^{\alpha}(A)\right) \odot \tau^{\beta}(B) \\
& \subseteq \bigcup_{\alpha<\lambda}\left(\tau^{\alpha}(A) \odot \tau^{\beta}(B)\right) \\
& \subseteq\langle A \odot B\rangle
\end{aligned}
$$

and similarly,

$$
\tau^{\alpha}(A) \odot \tau^{\lambda}(B) \subseteq\langle A \odot B\rangle
$$

Thus

$$
\begin{aligned}
\langle\langle A\rangle \odot\langle B\rangle\rangle & =\left\langle\tau^{*}(A) \odot \tau^{*}(B)\right\rangle \\
& \subseteq\langle A \odot B\rangle .
\end{aligned}
$$


Now we define a closed semiring $\mathbf{C K}$ as follows. The elements of $\mathbf{C K}$ will be the countably generated ${ }^{*}$-ideals of $\mathcal{K}$. For any countable set of countably generated ${ }^{*}$-ideals $I_{n}$, define

$$
\sum_{n} I_{n}=\left\langle\bigcup_{n} I_{n}\right\rangle
$$

This ideal is countably generated, since if $A_{n}$ is countable and generates $I_{n}$ for $n \geq 0$, then $\bigcup_{n} A_{n}$ is countable and generates $\sum_{n} I_{n}$. The operator $\sum$ is associative, commutative, and idempotent, since $\bigcup$ is.

For any pair of elements $I, J$, define

$$
I \cdot J=\langle I \odot J\rangle .
$$

This ideal is countably generated if $I$ and $J$ are, since

$$
\begin{aligned}
\langle A\rangle \cdot\langle B\rangle & =\langle\langle A\rangle \odot\langle B\rangle\rangle \\
& =\langle A \odot B\rangle
\end{aligned}
$$

by Lemma 3 , and $A \odot B$ is countable if $A$ and $B$ are.

The ideal $\langle 0\rangle=\{0\}$ is included in every ideal and is thus an additive identity. It is also a multiplicative annihilator:

$$
\begin{aligned}
\langle 0\rangle \cdot I & =\langle\langle 0\rangle \odot I\rangle \\
& =\langle\{0\} \odot I\rangle \\
& =\langle 0\rangle .
\end{aligned}
$$

The ideal $\langle 1\rangle$ is a multiplicative identity:

$$
\begin{aligned}
\langle 1\rangle \cdot I & =\langle\langle 1\rangle \odot I\rangle \\
& =\langle\{1\} \odot I\rangle \text { by Lemma } 3 \\
& =\langle I\rangle \\
& =I .
\end{aligned}
$$

Finally, the distributive laws hold:

$$
\begin{aligned}
I \cdot \sum_{n} J_{n} & =\left\langle I \odot \sum_{n} J_{n}\right\rangle \\
& =\left\langle I \odot\left\langle\bigcup J_{n}\right\rangle\right\rangle \\
& =\left\langle I \odot \bigcup_{n} J_{n}\right\rangle \text { by Lemma } 3 \\
& =\left\langle\bigcup_{n} I \odot J_{n}\right\rangle
\end{aligned}
$$




$$
\begin{aligned}
& =\left\langle\bigcup_{n}\left\langle I \odot J_{n}\right\rangle\right\rangle \\
& =\left\langle\bigcup_{n} I \cdot J_{n}\right\rangle \\
& =\sum_{n} I \cdot J_{n},
\end{aligned}
$$

and symmetrically.

Using the fact $\langle x\rangle=\{x\} \downarrow$, it is straightforward to verify that the map

$$
x \mapsto\langle x\rangle
$$

is one-to-one and preserves $+, \cdot,{ }^{*}, 0$, and 1 , thus constitutes an isomorphic embedding of $\mathcal{K}$ into the ${ }^{*}$-continuous Kleene algebra $\mathbf{K C K}$. This map will be the unit of our adjunction.

If all references to countability are removed from the foregoing construction, we get an embedding of $\mathcal{K}$ into an $\mathbf{S}$-algebra consisting of (not necessarily countably generated)

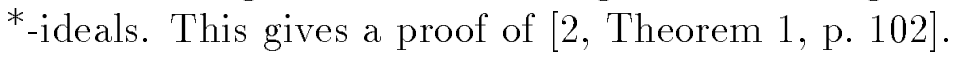

We now wish to establish an adjunction between $\mathbf{K A} \mathbf{A}^{*}$ and $\mathbf{C S}$. The first matter at hand is to establish a bijection between the homsets $\mathcal{K} \rightarrow \mathbf{K C}$ and $\mathbf{C K} \rightarrow \mathcal{C}$ for a ${ }^{*}$-continuous Kleene algebra $\mathcal{K}$ and closed semiring $\mathcal{C}$.

Let $g: \mathcal{K} \rightarrow \mathbf{K C}$ a Kleene algebra morphism. For a subset $A$ of $\mathcal{K}$, define

$$
g[A]=\{g(x) \mid x \in A\} .
$$

Lemma 4 For any $A \subseteq \mathcal{K}$ and ordinal $\alpha$, the following hold:

$$
\begin{aligned}
g[\tau(A)] & \subseteq \tau(g[A]) \\
g\left[\tau^{\alpha}(A)\right] & \subseteq \tau^{\alpha}(g[A]) \\
g[\langle A\rangle] & \subseteq\langle g[A]\rangle \\
\langle g[\langle A\rangle]\rangle & =\langle g[A]\rangle .
\end{aligned}
$$

Proof. To prove the first statement, note that

$$
\begin{aligned}
g[A \oplus A] & =\{g(x)+g(y) \mid x, y \in A\} \\
& =g[A] \oplus g[A] \\
g[A \downarrow] & =\{g(y) \mid \exists x \in A y \leq x\} \\
& \subseteq\{g(y) \mid \exists x \in A g(y) \leq g(x)\} \\
& \subseteq g[A] \downarrow
\end{aligned}
$$




$$
\begin{aligned}
g\left[A_{*}\right] & =\left\{g\left(x y^{*} z\right) \mid x y^{n} z \in A, n \geq 0\right\} \\
& =\left\{g(x) g(y)^{*} g(z) \mid x y^{n} z \in A, n \geq 0\right\} \\
& \subseteq\left\{g(x) g(y)^{*} g(z) \mid g(x) g(y)^{n} g(z) \in g[A], n \geq 0\right\} \\
& \subseteq g[A]_{*} .
\end{aligned}
$$

Then

$$
\begin{aligned}
g[\tau(A)] & =g\left[(A \oplus A) \cup A \downarrow \cup A_{*}\right] \\
& =g[A \oplus A] \cup g[A \downarrow] \cup g\left[A_{*}\right] \\
& \subseteq(g[A] \oplus g[A]) \cup g[A] \downarrow \cup g[A]_{*} \\
& =\tau(g[A]) .
\end{aligned}
$$

The second statement follows from the first by transfinite induction.

The third follows from the second by taking $\alpha$ sufficiently large.

The inclusion $\subseteq$ of the last statement follows from the third statement, and the inclusion $\supseteq$ follows from the monotonicity of $g$ and \langle\rangle .

Lemma 5 If $I \in \mathbf{C K}$, then $\sum g[I]$ exists and is equal to $\sum g[A]$ for any generating set $A$ of $I$.

Proof. By Lemma 4,

$$
\langle g[I]\rangle=\langle g[\langle A\rangle]\rangle=\langle g[A]\rangle
$$

for any generating set $A$ of $I$. In particular, since $I \in \mathrm{CK}, I$ has a countable generating set $B$, and since $\mathbf{C K}$ is closed under countable suprema, $\sum g[B]$ exists. Then

$$
\langle g[A]\rangle=\langle g[B]\rangle \subseteq\left\langle\sum g[B]\right\rangle=\left(\sum g[B]\right) \downarrow,
$$

thus $\sum g[B]$ is an upper bound for $g[A]$. For any other upper bound $y$,

$$
g[B] \subseteq\langle g[A]\rangle \subseteq\langle y\rangle,
$$

thus $\sum g[B] \leq y$.

There is a subtlety here: it is tempting to conclude that $\langle g[I]\rangle=\left\langle\sum g[I]\right\rangle$ in Lemma 5 . This appears not to be true in general.

Now define the map $\bar{g}: \mathbf{C K} \rightarrow \mathcal{C}$ by

$$
\bar{g}(I)=\sum g[I] .
$$

This is well defined by Lemma 5 .

We have not yet specified the action of the functor $\mathbf{C}$ on morphisms of $\mathbf{K} \mathbf{A}^{*}$. This can be be derived from the adjunction. For any pair $\mathcal{K}, \mathcal{K}^{\prime}$ of ${ }^{*}$-continuous Kleene algebras and Kleene algebra morphism $h: \mathcal{K} \rightarrow \mathcal{K}^{\prime}$, define

$$
\mathrm{C} h=\overline{\langle\rangle \circ h} \text {. }
$$


Theorem 6 The map $\bar{g}$ is an $\omega$-continuous semiring morphism. The construction $g \mapsto \bar{g}$ gives a natural bijection between the homsets $\mathcal{K} \rightarrow \mathbf{K C}$ and $\mathbf{C K} \rightarrow \mathcal{C}$.

Proof. To show that $\bar{g}$ is an $\omega$-continuous semiring morphism, we must show that $\sum, \cdot$, 0 , and 1 are preserved.

$$
\begin{aligned}
\bar{g}\left(\sum_{n} I_{n}\right) & =\sum g\left[\sum_{n} I_{n}\right] \\
& =\sum g\left[\left\langle\bigcup_{n} I_{n}\right\rangle\right] \\
& =\sum g\left[\bigcup_{n} I_{n}\right] \text { by Lemma } 5 \\
& =\sum \bigcup_{n} g\left[I_{n}\right] \\
& =\sum_{n} g\left[\left[I_{n}\right]\right. \\
& =\sum_{n} \bar{g}\left(I_{n}\right) \\
\bar{g}(I \cdot J) & =\sum g[I \cdot J] \\
& =\sum g[\langle I \odot J\rangle] \\
& =\sum g[I \odot J] \text { by Lemma } 5 \\
& =\sum g[I] \odot g[J] \\
& =\sum g[I] \cdot \sum g[J] \text { by infinite distributivity } \\
& =\bar{g}(I) \cdot \bar{g}(J) .
\end{aligned}
$$

That $\bar{g}$ preserves 0 and 1 is straightforward to verify.

To show that $g \mapsto \bar{g}$ is a bijection, define the map $h \mapsto h^{\prime}$ for $h: \mathrm{CK} \rightarrow \mathcal{C}$ by:

$$
h^{\prime}(x)=h(\langle x\rangle) .
$$

Straightforward arrow-chasing shows that ${ }^{-}$and ' are inverses.

Strictly speaking, in order to establish an adjunction, we must also check a naturality condition that ensures that the bijection given by ${ }^{-}$and ' interacts nicely with morphisms in $\mathbf{K A}^{*}$ and $\mathbf{C S}$; see [9]. Specifically, we must show that for all morphisms $h: \mathcal{K}^{\prime} \rightarrow \mathcal{K}$, $g: \mathcal{K} \rightarrow \mathbf{K C}$, and $f: \mathcal{C} \rightarrow \mathcal{C}^{\prime}$

$$
\overline{\mathbf{K} f \circ g \circ h}=f \circ \bar{g} \circ \mathbf{C} h .
$$

Again, this can be verified by straightforward arrow-chasing, which we leave to the reader. 


\subsection{Closed semirings and S-algebras}

The other half of the factorization of Conway's construction embeds an arbitrary closed semiring into an $\mathbf{S}$-algebra. In comparison to the construction of previous section, this construction is much less interesting. We give the main construction and omit formal details.

Recall that closed semirings and $\mathbf{S}$-algebras are both idempotent semirings with an infinite summation operator $\sum$ satisfying infinitary associativity, commutativity, idempotence, and distributivity laws. The only difference is that closed semirings allow only countable sums, whereas $\mathbf{S}$-algebras allow arbitrary sums. Morphisms of closed semirings are the $\omega$-continuous semiring morphisms and those of $\mathbf{S}$-algebras are the continuous semiring morphisms.

To construct an embedding of a given closed semiring $\mathcal{C}$ in an $\mathbf{S}$-algebra $\mathbf{S C}$, we complete $\mathcal{C}$ by ideals. An ideal is a subset $A \subseteq \mathcal{C}$ such that

- $A$ is nonempty

- $A$ is closed under countable sum

- $A$ is closed downward under $\leq$.

Take $\mathbf{S C}$ to be the set of ideals of $\mathcal{C}$ with the following operations:

$$
\begin{aligned}
\sum_{\alpha} I_{\alpha} & =\left\langle\bigcup_{\alpha} I_{\alpha}\right\rangle \\
I \cdot J & =\langle I \odot J\rangle \\
0 & =\langle 0\rangle \\
1 & =\langle 1\rangle
\end{aligned}
$$

where $\odot$ is defined as in $\S 4.1$.

The arguments from here on are quite analogous to those of $\S 4.1$.

\section{The universal Horn theory of regular events}

The universal Horn theory of a class of structures is the set of universally quantified equational implications of the form

$$
\alpha_{1}=\beta_{1} \wedge \cdots \wedge \alpha_{n}=\beta_{n} \rightarrow \alpha=\beta
$$

true in all structures in the class. In this section, we give a brief argument showing that the axioms for Kleene algebra given in $\S 2$, which characterize the universal Horn theory of Kleene algebras and the equational theory of $\mathbf{R e g}_{\Sigma}$, do not characterize the universal Horn theory of $\mathbf{R e g}_{\Sigma}$. This refutes a conjecture of Conway [2, p. 103]. 
Consider the equational implication

$$
a^{2}=1 \rightarrow a=1
$$

This formula is true in $\boldsymbol{R e g}_{\Sigma}$ for all values of $a$; in other words, for any regular event $A$, if $A^{2}=\{\epsilon\}$ then $A=\{\epsilon\}$. However, this formula is not a theorem of Kleene algebra, since there exists a Kleene algebra in which it fails: interpret $a$ as the matrix

$$
\left[\begin{array}{ll}
0 & 1 \\
1 & 0
\end{array}\right]
$$

in the Kleene algebra of $2 \times 2$ matrices over $\boldsymbol{R e g}_{\Sigma}$. This algebra was shown to be a Kleene algebra in [6].

It is an open problem to give a complete axiomatization of the universal Horn theory of the regular events.

\section{Right-handed Kleene algebras are not necessarily left-handed}

Recall the right- and left-handed Kleene algebra rules

$$
\begin{aligned}
& a x \leq x \rightarrow a^{*} x \leq x \\
& x a \leq x \rightarrow x a^{*} \leq x
\end{aligned}
$$

In [15], Pratt asks whether these two rules are equivalent, and conjectures that they are not. His conjecture actually refers to the more specific case of Boolean monoids, in which $\leq$ comes from a Boolean algebra. However, the question makes sense in the more general context of Kleene algebras. In this section we construct a right-handed Kleene algebra that is not left handed; this answers Pratt's question in the more general case of Kleene algebras and provides evidence in favor of his conjecture. The conjecture as stated in [15] is still open.

Consider the set $\mathcal{F}$ of strict, finitely additive set functions $f: \mathcal{P}(X) \rightarrow \mathcal{P}(X)$ for any infinite set $X$; that is, functions $f$ for which

$$
\begin{aligned}
f(A \cup B) & =f(A) \cup f(B) \\
f(\emptyset) & =\emptyset .
\end{aligned}
$$

Define

$$
\begin{aligned}
(f \cup g)(A) & =f(A) \cup g(A) \\
(f \circ g)(A) & =f(g(A))
\end{aligned}
$$




$$
\begin{aligned}
f^{0}(A) & =A \\
f^{\alpha+1}(A) & =f\left(f^{\alpha}(A)\right) \\
f^{\lambda}(A) & =\bigcup_{\alpha<\lambda} f^{\alpha}(A), \quad \lambda \text { a limit ordinal } \\
f^{*}(A) & =\bigcup_{\alpha} f^{\alpha}(A) \\
0(A) & =\emptyset \\
1(A) & =A .
\end{aligned}
$$

Note that finite additivity implies monotonicity.

Proposition 7 The structure

$$
\left(\mathcal{F}, \cup, \cdot{ }^{*}, 0,1\right)
$$

is a right-handed Kleene algebra but not a left-handed Kleene algebra.

Proof. To show that $\mathcal{F}$ is a right-handed Kleene algebra, we need to verify all the Kleene algebra axioms except (4). The additive associativity, commutativity, idempotence, and identity laws hold since they hold for set union. Function composition is surely associative with two-sided identity 1 . The function 0 is a left annihilator by definition and a right annihilator because of strictness of functions in $\mathcal{F}$. The left distributivity law holds by definition of $U$ and $o$, and the right distributivity law holds because of finite additivity of functions in $\mathcal{F}$. Thus $\mathcal{F}$ is an idempotent semiring.

The axioms (1-3) for ${ }^{*}$ can be verified by transfinite induction. Let us verify the righthanded rule (3). Suppose $f \circ g \leq g$. Then for any $A \subseteq X, f(g(A)) \subseteq g(A)$. We have

$$
\begin{aligned}
f^{0}(g(A)) & =g(A) \\
f^{\alpha+1}(g(A)) & =f\left(f^{\alpha}(g(A))\right) \\
& \subseteq f(g(A)) \text { by induction hypothesis and monotonicity of } f \\
& \subseteq g(A) \\
f^{\lambda}(g(A)) & =\bigcup_{\alpha<\lambda} f^{\alpha}(g(A)) \\
& \subseteq \bigcup_{\alpha<\lambda} g(A) \\
& =g(A), \lambda \text { a limit ordinal } \\
& =\bigcup_{\alpha} f^{\alpha}(g(A)) \\
f^{*}(g(A)) & \subseteq g(A) .
\end{aligned}
$$


All we need to show that (4) is not satisfied is the existence of a strict, finitely additive function $g$ that is not $\omega$ continuous, i.e. such that

$$
g^{\omega+1} \not g^{\omega}
$$

Then taking $f=g^{\omega}$, we have

$$
f \circ g=g^{\omega} \circ g \leq g^{\omega}=f
$$

but

$$
\begin{aligned}
f \circ g^{*} \leq f & \rightarrow g^{\omega} \circ g^{*} \leq g^{\omega} \\
& \rightarrow g^{*} \leq g^{\omega} \\
& \rightarrow g^{\omega+1} \leq g^{\omega}
\end{aligned}
$$

contradicting (6). There are many $g$ that satisfy (6); for example, pick out a countable proper subset $Y$ of $X$ and call them the natural numbers; then define

$$
g(A)= \begin{cases}A \cup\{x+1 \mid x \in A\}, & \text { if } A \text { is a finite subset of } Y \\ X, & \text { otherwise. }\end{cases}
$$

Then $g^{\omega}(\{0\})=Y$ but $g^{\omega+1}(\{0\})=g(Y)=X$.

\section{Acknowledgments}

I am grateful to John Horton Conway, Vaughan Pratt, and Dana Scott for their valuable comments.

\section{References}

[1] Alfred V. Aho, John E. Hopcroft, and Jeffrey D. Ullman. The Design and Analysis of Computer Algorithms. Addison-Wesley, 1975.

[2] John Horton Conway. Regular Algebra and Finite Machines. Chapman and Hall, London, 1971.

[3] John Horton Conway, personal communication, May 1990.

[4] Harel, D., First-Order Dynamic Logic. Lecture Notes in Computer Science 68, SpringerVerlag, 1979. 
[5] Dexter Kozen, "On induction vs. *-continuity," Proc. Workshop on Logics of Programs 1981, Spring-Verlag Lect. Notes in Comput. Sci. 131, ed. Kozen, 1981, 167-176.

[6] Dexter Kozen, "A completeness theorem for Kleene algebras and the algebra of regular events," Cornell TR90-1123, May 1990.

[7] Werner Kuich, "The Kleene and Parikh Theorem in Complete Semirings," in: Proc. 14th Colloq. Automata, Languages, and Programming, ed. Ottmann, Springer-Verlag Lecture Notes in Computer Science 267, 1987, 212-225.

[8] Werner Kuich and Arto Salomaa. Semirings, Automata, and Languages. SpringerVerlag, Berlin, 1986.

[9] Saunders Mac Lane. Categories for the Working Mathematician. Springer-Verlag, 1971.

[10] Kurt Mehlhorn. Data Structures and Algorithms 2: Graph Algorithms and NP-Completeness. EATCS Monographs on Theoretical Computer Science, Springer-Verlag, 1984.

[11] K. C. Ng and A. Tarski, "Relation algebras with transitive closure," Abstract 742-02-09, Notices Amer. Math. Soc. 24 (1977), A29-A30.

[12] K. C. Ng. Relation Algebras with Transitive Closure. PhD Thesis, University of California, Berkeley, 1984.

[13] Vaughan Pratt, "Semantical Considerations on Floyd-Hoare Logic," Proc. 17th IEEE Symp. Found. Comput. Sci. 1976, 109-121.

[14] Vaughan Pratt, "Dynamic algebras and the nature of induction," Proc. 12th ACM Symp. on Theory of Computing, 1980, 22-28.

[15] Vaughan Pratt, "Dynamic algebras as a well-behaved fragment of relation algebras," in: D. Pigozzi, ed., Proc. Conf. on Algebra and Computer Science, Ames, Iowa, June 2-4, 1988; Springer-Verlag Lecture Notes in Computer Science, to appear.

[16] Arto Salomaa, "Two complete axiom systems for the algebra of regular events," $J$. Assoc. Comput. Mach. 13:1 (January, 1966), 158-169. 